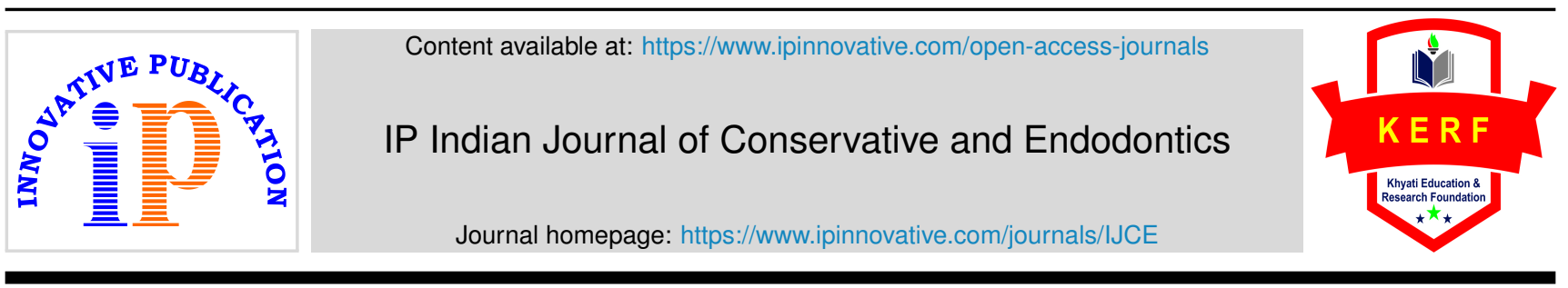

Original Research Article

\title{
An in-vitro comparative study of cyclic fatigue resistance of twisted files, Hyflex cm, Hyflex edm and Edgefile X3 after immersion in sodium hypochlorite and/or sterilization
}

\author{
Priya Sinha ${ }^{1}{ }^{*}$, Debaprasad Das ${ }^{1}$, Anirban Bhattacharyya ${ }^{1}$, Asim Bikash Maity ${ }^{1}$, \\ Trishagni Chaudhury ${ }^{1}$ \\ ${ }^{1}$ Dept. of Conservative Dentistry and Endodontics, Haldia Institute of Dental Sciences and Research, Haldia, West Bengal, India
}

\section{A R T I C L E I N F O}

Article history:

Received 21-12-2021

Accepted 22-01-2021

Available online 16-03-2021

\section{Keywords:}

Cyclic fatigue

controlled memory wire

Twisted File

Hyflex CM

Hyflex EDM

EdgeFile X3

\begin{abstract}
A B S T R A C T
Aim: The purpose of this study was to assess the effects of sodium hypochlorite $(\mathrm{NaOCl})$ immersion and sterilization on the cyclic fatigue resistance of four heat-treated nickel-titanium (NiTi) rotary instruments. Materials and Methods: Two hundred and forty new nickel-titanium endodontic files were tested for cyclic fatigue resistance in simulated canals. 25/.06 Twisted Files (Sybron Endo, Orange, CA, USA), 25/.06 Hyflex CM (Coltene Whaledent, Cuyahoga Falls, OH), 25/ Hyflex EDM (Coltene, Whaledent Pvt Ltd) and 25/.06 EdgeFile X3 (EdgeEndo, Albuquerque, NM, USA) files were divided into 4 subgroups ( $n=15)$ for each brand. Subgroup 1 (control group) included new instruments that were not immersed in $\mathrm{NaOCl}$ or subjected to autoclave sterilization. Subgroup 2 were composed of instruments dynamically immersed for 3 minutes in $5.25 \% \mathrm{NaOCl}$ solution. Subgroup 3 consisted of instruments that underwent 3 cycles of autoclave sterilization at $121^{\circ} \mathrm{C}$ under $15 \mathrm{psi}$ for 15 minutes. Subgroup 4 recruited instruments that received a cycle of both dynamic immersion in $\mathrm{NaOCl}$ solution for 3 minutes and 3 cycles of autoclave sterilization. All the files were rotated till fracture occurred. The means and standard deviations of the number of cycles to failure (NCF) were calculated and statistically analyzed using ANOVA with post-hoc Games Howell test. $(\mathrm{P}<.001)$.

Results: Comparison among the four investigated instruments indicated statistically significant difference of NCF $(\mathrm{P}>$.001). EdgeFile X3 files exhibited highest cyclic fatigue resistance, followed by Hyflex EDM files, Hyflex CM files and lowest resistance exhibited by Twisted Files in all the subgroups. There was also statistical significant difference $(\mathrm{P}<.001)$ when comparing the instruments in respect to the four treatment protocols (subgroups). Instruments undergoing dynamic immersion in $\mathrm{NaOCl}$ solution for 3 minutes followed by 3 cycles of autoclave sterilization did influence the cyclic fatigue of NiTi files tested, which showed a significant increase of cyclic fatigue resistance for all the four different heat-treated instruments when compared with the control group.

Conclusions: Within the limitations of this study, fracture resistance to cyclic fatigue was the highest for the EdgeFile X3 group followed by Hyflex EDM group, Hyflex CM group and lowest for Twisted File group in all the subgroups. Instruments undergoing dynamic immersion in $\mathrm{NaOCl}$ solution for 3 minutes followed by 3 cycles of autoclave sterilization did influence.

(C) This is an open access article distributed under the terms of the Creative Commons Attribution License (https://creativecommons.org/licenses/by/4.0/) which permits unrestricted use, distribution, and reproduction in any medium, provided the original author and source are credited.
\end{abstract}

\section{Introduction}

Nickel-titanium (NiTi) endodontic rotary files have been mostly utilized to shape curved root canals because of

\footnotetext{
* Corresponding author.

E-mail address: dr.psinha06@gmail.com (P. Sinha).
}

their superior flexibility. However, fracture of these NiTi instruments may occur without warning, even with brand new instruments, whereas fracture of stainless steel files is preceded by instrument distortion serving as a warning of impending fracture. ${ }^{1}$ File fracture might eventuate as a result of cyclic fatigue or torsional fatigue. ${ }^{2}$ Cyclic fatigue 
takes place because of repeated cycles of tension and compression, particularly when preparing curved canals, ultimately leading to fracture. Torsional fatigue takes place when tip of the file is locked to dentin walls while the shank continues to rotate. Prior studies have indicated that cyclic fatigue is the significant reason of file fracture. ${ }^{3}$

In recent years, countless innovative thermomechanical processing and proprietary manufacturing techniques have optimized the microstructure and the flexibility of $\mathrm{NiTi}$ alloys. ${ }^{4}$ The innovative enhancement behind the heat treatment process is the raised Af (Austenite finish) temperature of the alloy. If Af is higher than the body temperature, the file will be in a mixed martensitic state, Rphase and austenitic structure. ${ }^{5}$ Therefore, heat treated files demonstrated a significant increase in flexibility and flexural fatigue resistance in intra-canal temperature.

A unique rotary NiTi file system with R-phase heat treatment technology has been introduced in the market by SybronEndo, known as Twisted File (TF; SybronEndo, Orange, CA, USA). The manufacturers claim the three new design methods of this process: R-phase heat treatment, twisting of the metal and special surface conditioning, significantly increase instruments resistance to cyclic fatigue and flexibility. ${ }^{6}$ Hyflex CM files (Coltene Whaledent Pvt. Ltd) are made up of controlled memory wire manufactured using a special thermomechanical process, making the files extremely flexible. They exhibit a lower percent by weight of nickel (52 Ni \% wt) than the common (54.5-57) $\mathrm{Ni} \%$ wt of the great majority of commercially available superelastic (SE) NiTi rotary instruments. ${ }^{7}$ Hyflex EDM files (Coltene Whaledent Pvt. Ltd) are one of the first endodontic instruments, made of controlled memory wire with electric discharge machining (EDM) technology. ${ }^{8}$ EdgeFile (EdgeEndo, Albuquerque, NM, USA) systems have been presented that was produced of an annealed heat treated NiTi alloy, brand named Fire-Wire. Files with different characteristics (X1, X3, X5, X7 and XR) are used in this system. EdgeFile X3 files are compatible with ProTaper and ProTaper Next (Dentsply Maillefer) rotary file system and can be employed in the same handpiece at the same speed and torque settings. ${ }^{9}$

An important factor that may weaken the fracture resistance of $\mathrm{NiTi}$ instruments is corrosion. Chemomechanical preparation, cleaning procedures, chemical disinfection, and sterilization cause corrosion of endodontic instruments. Endodontic treatment may directly involve contact with saliva, blood and infected pulp tissue, carrying micro-organisms from patient to patient. ${ }^{10}$ Cross-infection control is a major issue in dental care setting. Hence, the basic theorems of asepsis also apply to NiTi files whenever the instruments is re-used.

Sodium hypochlorite $(\mathrm{NaOCl})$ is the most commonly used irrigant in endodontic treatment. Pre-soaking the endodontic files in $\mathrm{NaOCl}$ is a proven method for complete removal of organic debris and disinfection. ${ }^{11}$ Although the strength of $\mathrm{NaOCl}$ solution must be balanced against potential damage to instruments by corrosion as it is highly corrosive to metals. Its active $\mathrm{ClO}^{-}$ions selectively removes nickel from the instrument surface and causes micropitting. ${ }^{12}$ These are the areas where stress gets concentrated, forming cracks and increasing the probability of fatigue failure. ${ }^{13}$

Because post-machining thermal treatment influences the properties of heat-treated alloys, heat generated by sterilization procedures could influence the mechanical properties of endodontic instruments. Repetitive use of files under clinical conditions requires autoclave sterilization after every use. Also, prearranged sets of selected files may not be used during the same appointment. As a result, the unused rotary files are also subjected to multiple autoclave cycles. Researchers reported that additional "heat treatment" during autoclave sterilization might improve the flexibility of files and would have a positive effect on the cyclic fatigue resistance. ${ }^{14}$

The resistance to cyclic fatigue of $\mathrm{NiTi}$ rotary instruments can be increased via improvements in the manufacturing process or by the use of new alloys with superior mechanical properties. However, there is no study on the influence of both autoclave sterilization and immersion in $\mathrm{NaOCl}$ on the cyclic fatigue resistance of $\mathrm{NiTi}$ instruments made by these four different heat-treated alloys (Twisted Files, Hyflex CM, Hyflex EDM and EdgeFile X3). Thus, the purpose of this study is to investigate the effect of $\mathrm{NaOCl}$ contact, repeated autoclave cycles and combination of both on the cyclic fatigue resistance on these instruments.

\section{Materials and Methods}

Total of two hundred and forty new files were evaluated in the present study

1. Group A: $60=$ Twisted File (25/.06) (Sybron Endo, Orange, CA, USA) $-27 \mathrm{~mm}$.

2. Group B: $60=$ Hyflex CM (25/.06) (Coltene Whaledent, Cuyahoga Falls, $\mathrm{OH})-25 \mathrm{~mm}$.

3. Group C: $60=$ Hyflex EDM One-File $(25 / \sim)($ Coltene Whaledent Pvt. Ltd.) - 25mm.

4. Group D: $60=$ EdgeFile X3 (25/.06) (EdgeEndo, Albuquerque, NM, USA) - 25mm.

Instruments from the same production lot were randomly assigned into 4 subgroups $(n=15)$ for each brand. Subgroup 1 (the control group) included new instruments that were not immersed in $\mathrm{NaOCl}$ or subjected to sterilization. Subgroup 2 was composed of instruments dynamically immersed for 3 minutes in $5.25 \% \mathrm{NaOCl}$ solution (CanalPro Coltene, Whaledent Pvt. Ltd.) at room temperature. All files were placed in small separate glass containers with the amount of $\mathrm{NaOCl}$ solution necessary to contact 16 $\mathrm{mm}$ of the instrument's length; dynamic immersion was 
allowed, activating the endodontic instruments with a 16:1 reduction handpiece (CanalPro CL2 Endo motor; Coltene, Whaledent Pvt Ltd) at $500 \mathrm{rpm}$. Immediately after removal from the solutions, all files were rinsed with distilled water (Pure Lab, Bengal Biotech and Research) to neutralize the effect of $\mathrm{NaOCl}$, dried, registered with an identification number, and stored in glass vials. Subgroup 3 consisted of instruments autoclaved only 3 times. They were subjected to 3 cycles of autoclave (IDS Denmed, Sun Medical Front Loading Class 'N') and each cycle was performed at a temperature of $121^{\circ} \mathrm{C}$ for 15 minutes under $15 \mathrm{psi}$. Each file was placed in a separate endodontic sponge and packaged singularly for sterilization in pouches (GreenGuava, Lot No. 712120, EN 868-5). Instruments were allowed to cool to room temperature after sterilization. Sponges were removed from sterilization packaging and repackaged singularly in pouches before the subsequent autoclave cycles. No additional cleaning or surface treatment procedures were performed on the files before, during, or after sterilization. Subgroup 4 recruited instruments that were first dynamically immersed in $\mathrm{NaOCl}$ for 3 minutes followed by 3 cycles of autoclave sterilization.

Instruments of all subgroups of each brand were then subjected to cyclic fatigue testing using a customized stainless steel metallic block with artificial canals. $80 \mathrm{~mm}$ x $30 \mathrm{~mm}$ x $10 \mathrm{~mm}$ stainless steel metal block with 4 suitable artificial canals of $2 \mathrm{~mm}$ wide with $75^{\circ}$ angle of curvature, 5 $\mathrm{mm}$ radius of curvature to the center and an overall length of $17 \mathrm{~mm}$ were constructed. This metal block was constructed according to the method of Schneider. ${ }^{15}$ Cyclic fatigue tests were performed by rotating each instrument attached to in continuous rotation at $500 \mathrm{rpm}$. Torque was set at maximum level as recommended by the manufacturer of respective instruments (Twisted Files $=2 \mathrm{~N}-\mathrm{cm}$, Hyflex $\mathrm{CM}=2.5$ $\mathrm{N}-\mathrm{cm}$, Hyflex EDM = $2.5 \mathrm{~N}-\mathrm{cm}$, EdgeFile X3 = $3 \mathrm{~N}-\mathrm{cm}$ ). The stopper of the files were set at a constant length of 17 $\mathrm{mm}$ from the tip so that the bend on the file occurred at the level of D5. Artificial canals were covered with a thin glass slab ( 3 inch $\times 3$ inch) to prevent the instrument from slipping out and to allow observation of the instruments. To reduce friction between the instrument and metal canal walls, a synthetic oil (Turbo X Spray Plus) was applied for lubrication of mechanical parts. The device enabled the instrument to rotate freely within the artificial canal at a constant pressure.

All instruments were rotated until fracture occurred. The time was noted with a stopwatch which was stopped as fracture was detected visually and/or audibly.

\section{Calculation}

The number of cycles to failure (NCF) for each instrument was calculated by multiplying the number of rotations per minute (RPM) multiplied by total time taken for the instrument to fracture (minutes).
$\mathrm{NCF}=\mathrm{RPM} \times\{$ Total time taken for the instrument to fracture (secs)/60\}.

All data regarding the NCF are collected and recorded based on their respective instruments and treatment subgroups. They were statistically analysed using ANOVA with post-hoc Games-Howell test to assess significant differences among groups and subgroups $(\mathrm{P}<.001)$.

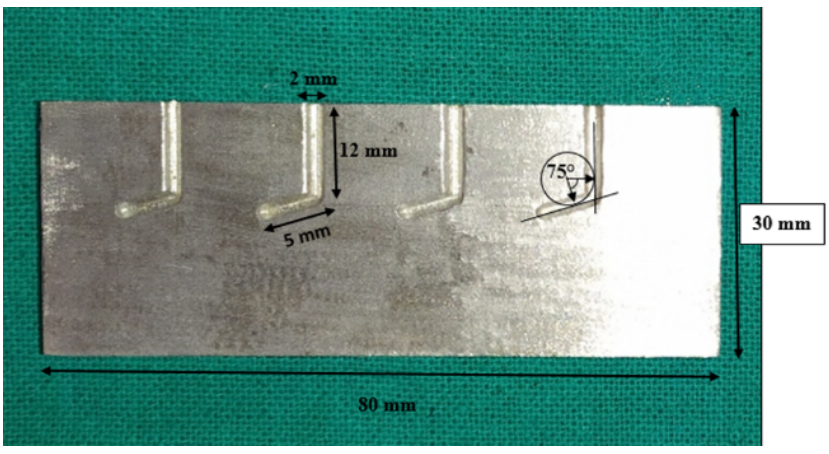

Fig. 1: Customized stainless steel metal block for cyclic fatigue testing

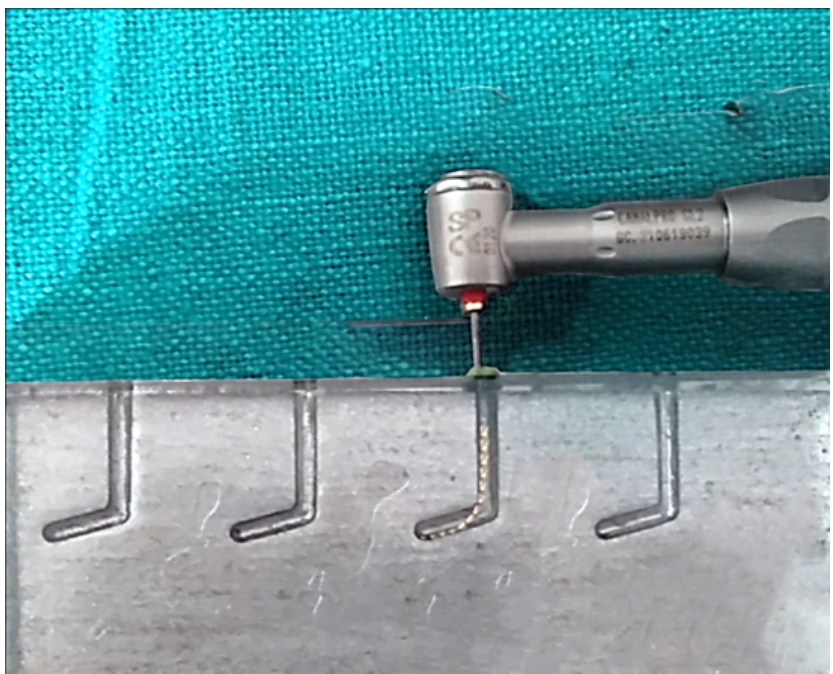

Fig. 2: Cyclic fatigue test performed

\subsection{Statistical analysis}

All the analysis was done using SPSS version 18. A pvalue of $<0.001$ was considered statistically significant. Comparison of mean NCF (number of cycles to failure) values were done using ANOVA with post-hoc Games Howell test.

\section{Results}

The means and standard deviations of NCF after the cyclic fatigue test for all the subgroups of investigated instruments 
Table 1: Comparison of NCF with respect to each treatment protocol (subgroups) among four different types of files.

\begin{tabular}{|c|c|c|c|c|c|c|c|c|c|c|}
\hline & \multicolumn{2}{|c|}{ Twisted File[A] } & \multicolumn{2}{|c|}{ Hyflex CM [B] } & \multicolumn{2}{|c|}{$\begin{array}{c}\text { Hyflex EDM } \\
{[C]}\end{array}$} & \multicolumn{2}{|c|}{ Edgefile X3 [D] } & \multirow[t]{2}{*}{ P-value } & \multirow[t]{2}{*}{$\begin{array}{c}\text { Post-hoc } \\
\text { test }\end{array}$} \\
\hline & Mean & SD & Mean & SD & Mean & SD & Mean & SD & & \\
\hline Subgroup 1 & 273.69 & 59.94 & 438.48 & 127.27 & 762.81 & 107.64 & 807.22 & 172.77 & $\begin{array}{c}<0.001 \\
\quad \text { Sig }\end{array}$ & $\mathrm{C}, \mathrm{D}>\mathrm{B}>\mathrm{A}$ \\
\hline Subgroup 2 & 255.94 & 35.83 & 833.89 & 256.55 & 720.84 & 98.67 & 1215.00 & 295.17 & $\begin{array}{c}<0.001 \\
\quad \text { Sig }\end{array}$ & $\mathrm{D}>\mathrm{C}, \mathrm{B}>\mathrm{A}$ \\
\hline Subgroup 3 & 186.32 & 94.23 & 690.94 & 231.02 & 729.96 & 84.12 & 1360.00 & 597.02 & $\begin{array}{c}<0.001 \\
\quad \text { Sig }\end{array}$ & $\mathrm{D}>\mathrm{C}, \mathrm{B}>\mathrm{A}$ \\
\hline Subgroup 4 & 359.53 & 75.77 & 778.98 & 233.68 & 919.80 & 222.98 & 1595.00 & 395.48 & $\begin{array}{c}<0.001 \\
\quad \text { Sig }\end{array}$ & $\mathrm{D}>\mathrm{C}, \mathrm{B}>\mathrm{A}$ \\
\hline
\end{tabular}

Table 2: Comparison of NCF with respect to each file group among four different treatment protocols (subgroups).

\begin{tabular}{lccccccccccc}
\hline & \multicolumn{2}{c}{ Subgroup 1 } & \multicolumn{4}{c}{ Subgroup 2 } & \multicolumn{2}{c}{ Subgroup 3 } & \multicolumn{2}{c}{ Subgroup 4 } & \multicolumn{2}{c}{ P- } & Post-hoc \\
& Mean & SD & Mean & SD & Mean & SD & Mean & SD & value & test \\
Twisted File & 273.69 & 59.94 & 255.94 & 35.83 & 186.32 & 94.23 & 359.53 & 75.77 & $<0.001$ & $4>1,2,3$ \\
Hyflex CM & 438.48 & 127.27 & 833.89 & 256.55 & 690.94 & 231.02 & 778.98 & 233.68 & $<0.001$ & $4>1,2,3$ \\
Hyflex EDM & 762.81 & 107.64 & 720.84 & 98.67 & 729.96 & 84.12 & 919.80 & 222.98 & 0.027 & $4>2,3$ & $2,3>1$ \\
Edgefile X3 & 807.22 & 172.77 & 1215.00 & 295.17 & 1360.00 & 597.02 & 1595.00 & 395.48 & $<0.001$ & 2 \\
& & & & & & & & & & $4>1,2$ \\
\hline
\end{tabular}

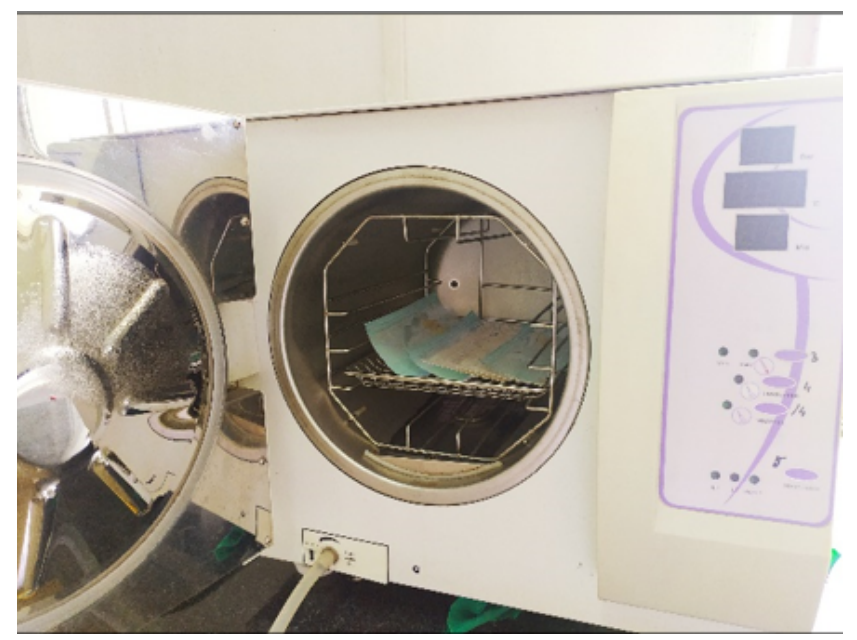

Fig. 3: Autoclave pouches with instruments placed inside the autoclave machine and autoclaved at $121^{\circ} \mathrm{c}$ under 15 psi for 15 minutes

are presented in Tables 1 and 2. Comparison of mean NCF among the different investigated instruments in respect to the four treatment protocol (subgroups) are presented in Charts 1 and 2.

The inferential analysis revealed that group D (EdgeFile $\mathrm{X} 3$ ) showed an increase in mean NCF values than group C (Hyflex EDM), group B (Hyflex CM) and group A (Twisted Files) in all the four subgroups as shown in Chart 1. The difference in values in mean NCF were statistically significant $(\mathrm{p}<0.001)$ in all subgroups. In subgroup 1(control group), post-hoc test showed that EdgeFile X3 and Hyflex EDM files had significantly higher

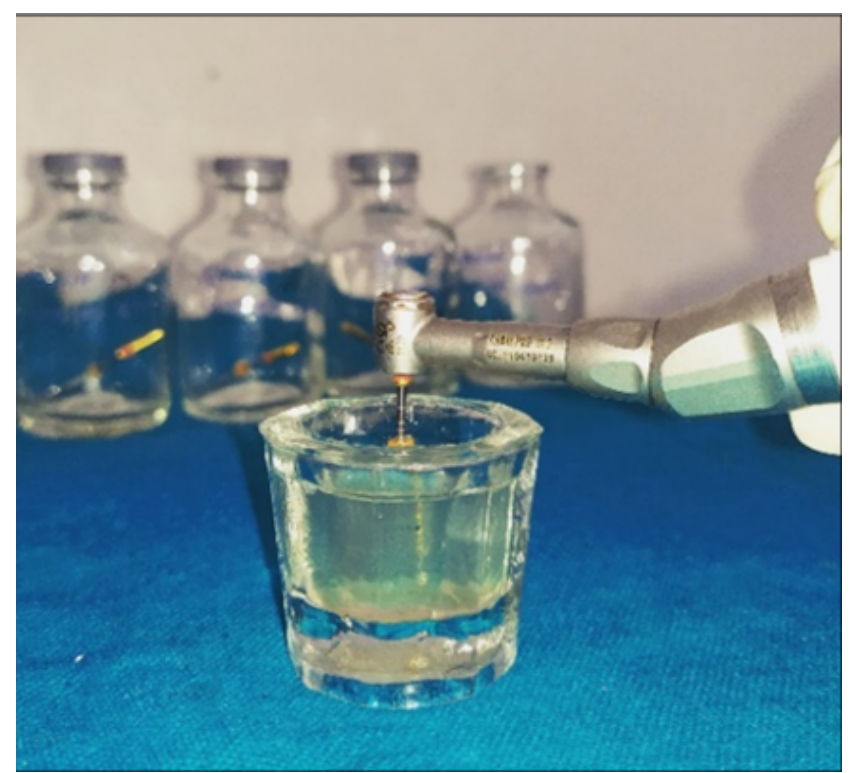

Fig. 4: Dynamic immersion in $5.25 \%$ sodium hypochlorite solution for 3 minutes

mean NCF $(\mathrm{p}<0.001)$ than Hyflex CM with least being in Twisted Files. No other significant differences were seen. In the rest three subgroups i.e. subgroup 2 (dynamic immersion in $5.25 \% \mathrm{NaOCl}$ solution for 3 minutes), subgroup 3 (3 cycles of autoclave sterilization) and subgroup 4 (dynamic immersion in $5.25 \% \mathrm{NaOCl}$ solution for 3 minutes and then 3 cycles of autoclave sterilization), EdgeFile X3 showed significantly higher mean NCF $(p<0.001)$ than Hyflex EDM and Hyflex CM with least being in Twisted Files. No other significant differences were seen. 
There was significant difference in the mean NCF with respect to different files tested with various treatment protocols $(\mathrm{p}<0.001)$. In Twisted Files and Hyflex CM files, Post-hoc test showed that subgroup 4 had significantly higher mean NCF $(\mathrm{p}<0.001)$ than subgroups 1,2 and 3. No other significant differences were seen. In Hyflex EDM, subgroup 4 showed significantly higher mean NCF $(\mathrm{p}<0.001)$ than subgroups 2 and 3 . No other significant differences were seen. In Edgefile X3, post-hoc test showed that subgroups 2 and 3 showed significantly higher mean $\mathrm{NCF}(\mathrm{p}<0.001)$ than subgroup 1. Also, subgroup 4 showed significantly higher mean $\mathrm{NCF}(\mathrm{p}<0.001)$ than subgroups 1 and 2. No other significant differences were seen.

\section{Discussion}

During clinical practice, NiTi instruments are more prone to fracture to cyclic fatigue as they are used several times. Cheung GS et al ${ }^{16}$ reported that upto $93 \%$ of instrument fractures have been classified as a result of cyclic fatigue. A retained instrument will further compromise root canal preparation, disinfection and root canal filling procedures. So the success rate of root canal treatment may decrease at least for teeth associated with periapical pathoses. ${ }^{1}$ It seems reasonable that any preventive strategy needs to take into account the mechanical and physical properties of the instruments used and should be based on sound and reliable results of the studies performed under conditions reflecting the clinical settings.

To better simulate the clinical conditions, different studies have used many methods to investigate in-vitro cyclic fatigue fracture resistance of NiTi rotary endodontic instruments. ${ }^{15}$ They include artificial canals that were constructed by bending glassor metalcylindrical tubes with different inner diameters and point of maximum curvature using different radii and angles of curvature. An artificial canal diameter of $2 \mathrm{~mm}$ has been selected for this study in order to have a loose fit of the tested instruments. Most commonly, a radius of $5 \mathrm{~mm}$ has been described and the centre of curvature are positioned $5 \mathrm{~mm}$ from the instrument tip. This model, as used in many studies, generates the highest flexural stress at the centre of the curve. ${ }^{17}$ Reducing the radius results in a reduced lifespan of NiTi instruments. A study by Plotino et al reported that artificial canals have been constructed with different angles of curvature ranging from $30^{\circ}-90^{\circ}$ simulating the severity of the curvature of the root canals. ${ }^{18}$ The usual method of measuring canal curvature is by Schneider method (1971). Each successive increase in angle of curvature resulted in significantly decreased NCF. In the present study, $75^{\circ}$ angle of curvature has been chosen in order to keep this parameter within the range based on the studies where artificial canals of $70^{\circ}$ and $90^{\circ}$ angles of curvature. ${ }^{19,20}$

$\mathrm{NiTi}$ alloys used for manufacturing of the endodontic instruments contain approximately $56 \%$ (wt) of nickel and
$44 \%$ (wt) of titanium. The composition of the NiTi alloy, especially the nickel content, has a great influence on the transformation temperatures. It has been reported that the phase transformation temperature shifts $12^{\circ} \mathrm{C}$ toward a lower temperature when the nickel atom content of a Ni-rich NiTi alloy increases by $0.1 \% .^{21}$ Change in environmental temperature affect the mechanical properties of the files. Increase in temperature improved the cyclic fatigue resistance. ${ }^{22}$ Heat treatment of NiTi to about 450$550^{\circ} \mathrm{C}$ releases the internal stresses and reduces the defects of the crystal lattice by arranging the atoms.

Although Twisted Files consist mainly of austenite in the oral environment, the bending load values in elastic and superelastic ranges are lower for Twisted Files than those of conventional Superelatic (SE) NiTi files. Hyflex $\mathrm{CM}$ files have a lower nickel content (52\% wt.), as compared to other NiTi files. To incorporate extreme flexibility, these files undergo special thermo-mechanical treatment that also improves their shape memory effect. Consequently, they do not have the rebound effect after unloading, and their original shape is restored after heat application or autoclaving procedure. The austenite finish temperature of Hyflex $\mathrm{CM}$ is $47-55^{\circ} \mathrm{C}$, which is above the intracanal temperature. ${ }^{23}$ The transformation temperatures of Hyflex EDM are found to be higher compared to Hyflex CM. According to the X-ray diffraction analysis, Hyflex EDM consists of martensite and substantial amounts of Rphase, whereas Hyflex $\mathrm{CM}$ has a mixture of martensite and austenite phases. ${ }^{24}$ The lack of austenitic phase in Hyflex EDM could be due to its raised austenitic start temperature (as $\sim 42^{\circ} \mathrm{C}$ ) that prevents the formation of austenite at room or body temperature. ${ }^{23}$ Hyflex EDM still has a higher hardness than conventional CM wires, despite absence of or reduced austenitic phase, thus substantiating the hardening effect of EDM. In contrast with these findings, the remarkable irregularities observed on the surface of HyFlex EDM did not compromise the instruments integrity after multiple uses. ${ }^{25}$ This is particularly unexpected since both EDM and CM files are manufactured with the same controlled memory wire and such great improvement is likely to be related to the EDM manufacturing process. EdgeFile has recently been launched as a constant tapered file system. According to the manufacturer, this annealed heat-treated Firewire NiTi provides significant flexibility. Heat treatment process as cryogenically tempered may have increased resistance to cyclic fatigue of EdgeFile X3 files.

Hence, Twisted Files, Hyflex CM, Hyflex EDM and EdgeFile X3 have been selected for the present study to compare their cyclic fatigue resistance. Additionally, all these four heat treated rotary NITi instruments have an operational speed of $500 \mathrm{rpm}$ as mentioned by their manufacturers. In the present study, EdgeFile X3 files exhibited higher cyclic fatigue resistance, followed by Hyflex EDM, Hyflex CM and Twisted Files, and this 
could be attributed to the differences in their metallurgical properties and their unique surface treatments.

To remain within a realistic time-frame of clinical practice, 3 minutes has been selected as the contact time of $\mathrm{NaOCl}$ solution with the instrument. According to the present study, $\mathrm{NaOCl}$ treatment alone did not alter nor affect the NCF of the NiTi instruments tested. This result confirmed that the new metal treatment on the heat treated instruments probably did not allow the corrosive effect by the sodium hypochlorite solution. The result of this study is in sync with a study by Pedulla et al where he reported that static or dynamic immersion in $5 \% \mathrm{NaOCl}$ did not significantly reduce the cyclic fatigue resistance of Twisted Files where test were performed on MTwo, Revo S SU, and Twisted Files systems. ${ }^{26}$ A significant decrease in cyclic fatigue resistance of ProFile \& $\mathrm{RaCe}$ instruments was observed in a study by Peters et al., after immersion in a heated $\mathrm{NaOCl}$ solution $\left(60^{\circ} \mathrm{C}\right)$ for 1 hour. ${ }^{27} \mathrm{O}$ 'Hoy et al. reported corrosion of $\mathrm{NiTi}$ instruments after immersion in $\mathrm{NaOCl}$ solution overnight. ${ }^{28}$ But this condition does not simulate the realistic time-frame of the root canal procedure.

The number of times that rotary instruments can be reused is suggested to vary between 1 and 10, depending on the canal conditions and the type of instrument usage. ${ }^{29}$ Clinicians often recycle NiTi files owing to economic considerations. In the present study, reuse for 3 times was simulated by three cycles of autoclave sterilization. Because post-machining thermal treatment influences the properties of heat-treated alloys, heat generated by sterilization procedures could influence the mechanical properties of endodontic instruments. In the present study, three cycles of autoclaving have not influenced the cyclic fatigue resistance of the four different tested instruments. Some authors reported no effect of autoclave sterilization on the fatigue resistance of NiTi files, others have shown a higheror lower mean NCF when exposed to both dry heat and autoclave sterilization. ${ }^{30}$ The explanation for these undesirable effects of sterilization may be that sterilized instruments undergo changes on their outer surfaces and in the chemical composition of the surface layer. Since, no effect on the NCF has been noticed after three cycles of autoclaving, it is evident that autoclaving can be performed upto three cycles without affecting the cyclic fatigue resistance of the tested instruments.

There has been a significantly increase in the NCF for all the four heat-treated files after immersion in sodium hypochlorite solution for 3 minutes followed by 3 cycles of autoclave sterilization. Though there has been an increase in $\mathrm{NCF}$ for all the four heat-treated files, comparative analysis showed the highest fracture resistance for EdgeFile X3 files, followed by Hyflex EDM, Hyflex CM with the least fracture resistance being the Twisted Files. The reason behind this may be due to the different manufacturing processes and different designs of the instruments used here. Very few studies have been done on these instruments with the parameters used. Among them, none gave such a result. In a similar study by Bulem et al., there was no effect on the cyclic fatigue resistance of the tested instruments (ProFile, FlexMaster, MTwo and Twisted Files) after combined process of autoclaving and dynamic immersion in sodium hypochlorite solution. ${ }^{29}$ Another study by Pedulla et al. analyzing the combined effect of $\mathrm{NaOCl}$ immersion and autoclave sterilization on the cyclic fatigue resistance of Hyflex CM and Twisted Files, revealed that there was no influence on cyclic fatigue resistance of the tested instruments. ${ }^{30}$ These different findings could be because of the unique metallurgical and manufacturing characteristics of these four heat-treated instruments tested.

\section{Conclusion}

Within the limitations of this study, following conclusions are drawn:

EdgeFile X3 exhibited highest cyclic fatigue resistance, followed by Hyflex EDM, Hyflex CM and the least fracture resistance being the Twisted Files in conditions like, after dynamic immersion in $\mathrm{NaOCl}$ solution and/or after autoclave sterilization. Dynamic immersion in 5.25\% $\mathrm{NaOCl}$ solution for 3 minutes alone did not affect significantly the cyclic fatigue resistance of all four tested heat-treated NiTi files, when compared with the control group. Repeated three cycles of autoclave sterilization alone did not influence the cyclic fatigue resistance of all four tested heat-treated NiTi files tested, when compared with the control group. Immersion in sodium hypochlorite solution along with repeated cycles of sterilization did influence the cyclic fatigue of NiTi files tested, which showed a significant increase of cyclic fatigue resistance for all the four tested heat-treated files, when compared with the control group.

\section{Source of Funding}

No financial support was received for the work within this manuscript.

\section{Conflict of Interests}

The author declares that they do not have any conflict of interests.

\section{References}

1. Parashos P, Messer HH. Rotary NiTi Instrument Fracture and its Consequences. $J$ Endod. 2006;32(11):1031-43. do1:10.1016/j.joen.2006.06.008.

2. Adıgüzel M, Capar ID. Comparison of Cyclic Fatigue Resistance of WaveOne and WaveOne Gold Small, Primary, and Large Instruments. J Endod. 2017;43(4):623-7. đo1:10.1016/1.joen.2016.11.021.

3. Topcuoglu HS, Topcuoglu G. Cyclic fatigue resistance of Reciproc Blue and Reciproc Files in an s-shaped canal. $J$ Endod. 2017;43(10):1679-82. 
4. Gutmann JL, Gao Y. Alteration in the inherent metallic and surface properties of nickel-titanium root canal instruments to enhance performance, durability and safety: a focused review. Int Endod $J$. 2012;45(2):113-28. doi:10.1111/1.1365-2591.2011.01957.x.

5. Adıgüzel M, Öztekin F. Comparison of the resistance to cyclic fatigue of One Curve, One Shape, 2Shape and EdgeFile X3 files in simulated single and S-shaped (double) curvatures. Int Dent Res. 2020;10(2):559.

6. Pujari H. Stress distribution of new generation of Twisted Files in comparison with ProTaper: A finite element analysis. Saudi Endod J. 2013;3(2):65-9. 100:10.4103/1658-5984.118149.

7. Tabassum S, Zafar K, Umer F. Nickel-Titanium Rotary File Systems: What's New? Eur Endod J. 2019;3:111-7.

8. Pedullà E, Savio FL, Boninelli S, Plotino G, Grande NM, Rosa GL, et al. Torsional and Cyclic Fatigue Resistance of a New Nickel-Titanium Instrument Manufactured by Electrical Discharge Machining. $\quad J$ Endod. 2016;42(1):156-9. doi:10.1016/j.joen.2015.10.004.

9. https://edgeendo.com/wp-content/uploads/2015/08/DFU-EdgeFile-x3 .pdf.

10. Recommendations for the prevention of HIV transmission in health care settings. Morbidity and mortality weekly report. 1987;36:3-18.

11. Hand RE, Smith ML, Harrison JW. Analysis of the effect of dilution on the necrotic tissue dissolution property of sodium hypochlorite. $J$ Endod. 1978;4(2):60-4. doi:10.1016/s0099-2399(78)80255-6.

12. Shen Y, Qian W, Abtin H, Gao Y, Haapasalo M. Effect of Environment on Fatigue Failure of Controlled Memory Wire Nickel-Titanium Rotary Instruments. J Endod. 2012;38(3):376-80. do1:10.1016/].joen.2011.12.002

13. Haïkel Y, Serfaty R, Wilson P, Speisser JM, Allemann C. Mechanical properties of nickel-titanium endodontic instruments and the effect of sodium hypochlorite treatment. J Endod. 1998;24(11):731-5. doi:10.1016/s0099-2399(98)80163-5.

14. Hilt B, Cunningham C, Shen C, Richards N. Torsional Properties of Stainless-Steel and Nickel-Titanium Files After Multiple Autoclave Sterilizations. J Endod. 2000;26(2):76-80. 101:10.09/100004/700 200002000-00004.

15. Schneider SW. A comparison of canal preparations in straight and curved root canals. Oral Surg Oral Med Oral Pathol. 1971;32:271-5.

16. Cheung GSP, Peng B, Bian Z, Shen Y, Darvell BW. Defects in ProTaper S1 instruments after clinical use: fractographic examination. Int Endod J. 2005;38(11):802-9. 001:10.1111/.1365एक्या20050020x

17. Pedullà E, Lizio A, Scibilia M, Grande NM, Plotino G, Boninelli $\mathrm{S}$, et al. Cyclic fatigue resistance of two nickel-titanium rotary instruments in interrupted rotation. Int Endod J. 2017;50(2):194-201. doi:10.1111/iej.12609

18. Ray JJ, Kirkpatrick TC, Rutledge RE. Cyclic Fatigue of EndoSequence and K3 Rotary Files in a Dynamic Model. J Endod. 2007;33(12):1469-72. do1:10.1016/1.joen.2007.07.041.

19. Iacono F. Wear analysis and cyclic fatigue resistance of electro discharge machined NiTi rotary instruments. $G$ Italiano di Endodonzia. 2016;30:64-8.

20. Lopes HP, Elias CN, Vieira MV. Fatigue life of Reciproc and Mtwo instruments subjected to static and dynamic tests. J Endod. 2013;39:693-6.

21. Melton KN. Ni-Ti based shape memory alloys. Engineering Aspects of Shape Memory Alloys. London: Butterworth-Heinemann; 1990. p. $21-5$.
22. Cheung GSP, Darvell BW. Fatigue testing of a NiTi rotary instrument Part 2: fractographic analysis. Int Endod J. 2007;40(8):619-25. doi:10.1111/1.1365-2591.2007.01256.X.

23. Shen Y, Zhou H, Zheng Y, Campbell L, Peng B, Haapasalo M, et al. Metallurgical Characterization of Controlled Memory Wire Nickel-Titanium Rotary Instruments. J Endod. 2011;37(11):1566-71. do1:10.1016/].joen.2011.08.005.

24. Iacono F, Pirani C, Generali L, Bolelli G, Sassatelli P, Lusvarghi L, et al. Structural analysis of HyFlex EDM instruments. Int Endod $J$. 2017;50(3):303-13. doi:10.1111/1ej.12620.

25. Iacono F, Pirani C, Generali L, Sassatelli P, Nucci C, Gandolfi MG, et al. Wear analysis and cyclic fatigue resistance of electro discharge machined NiTi rotary instruments. Giornale Italiano di Endodonzia. 2016;30(1):64-8. [01:10.1016/1.g1en.2016.04.006

26. Pedullà E, Grande NM, Plotino G, Pappalardo A, Rapisarda E. Cyclic Fatigue Resistance of Three Different Nickel-Titanium Instruments after Immersion in Sodium Hypochlorite. J Endod. 2011;37(8):113942. doi:10.1016/j.joen.2011.04.008

27. Peters OA, Roehlike JO, Baumann MA. Effect of Immersion in Sodium Hypochlorite on Torque and Fatigue Resistance of Nickel-Titanium Instruments. $\quad J$ Endod. 2007;33(5):589-93. do1:10.1016/1.10en.2007.01007

28. O'Hoy PY, Messer HH, Palamara JEA. The effect of cleaning procedures on fracture properties and corrosion of NiTi files. Int Endod J. 2003;36(11):724-32. doi:10.1046/].1365ए5912003.00700त.

29. Bulem UK, Kececi AD, Guldas HE. Experimental evaluation of cyclic fatigue resistance of four different nickel-titanium instruments after immersion in sodium hypochlorite and/or sterilization. J Appl Oral Sci . 2013;21(6):505-10. 101:101590/16/4-715/20130083].

30. Pedullà E, Benites A, Rosa GL, Plotino G, Grande NM, Rapisarda E, et al. Cyclic Fatigue Resistance of Heattreated Nickel-titanium Instruments after Immersion in Sodium Hypochlorite and/or Sterilization. J Endod. 2018;44(4):648-53. do1:10.1016/].joen.2017.12.01].

\section{Author biography}

Priya Sinha, Post Graduate Trainee

Debaprasad Das, Professor and HOD

Anirban Bhattacharyya, Professor

Asim Bikash Maity, Professor

Trishagni Chaudhury, Assistant Professor

Cite this article: Sinha P, Das D, Bhattacharyya A, Maity AB,

Chaudhury T. An in-vitro comparative study of cyclic fatigue resistance of twisted files, Hyflex cm, Hyflex edm and Edgefile X3 after immersion in sodium hypochlorite and/or sterilization. IP Indian $J$ Conserv Endod 2021;6(1):30-36. 